\title{
DEGREE FORMULAS FOR A TOPOLOGICAL INVARIANT OF BIFURCATIONS OF FUNCTION-GERMS
}

\author{
Nicolas Dutertre
}

\section{Introduction}

Let $F=\left(f_{1}, \ldots, f_{k}\right):\left(\boldsymbol{K}^{n}, 0\right) \rightarrow\left(\boldsymbol{K}^{k}, 0\right)$, with $1 \leq k<n$ and $\boldsymbol{K}=\boldsymbol{C}$ or $\boldsymbol{K}=$ $\boldsymbol{R}$, be an analytic map-germ with an isolated singularity at the origin. Let $g$ : $\left(\boldsymbol{K}^{n}, 0\right) \rightarrow(\boldsymbol{K}, 0)$ be an analytic function-germ. We are interested in computing topological invariants associated to the mappings $F$ and $(F, g)$.

Let $B_{\varepsilon} \subset K^{n}$ be a small ball centered at the origin and let $\delta \in K^{k}$ be a small regular value of $F$. The Milnor fiber of $F$ is $F^{-1}(\delta) \cap B_{\varepsilon}$. If $k=1$, Milnor [Mi2] proved that $F^{-1}(\delta) \cap B_{\varepsilon}$ has the homotopy type of a bouquet of $\mu(n-1)$-spheres where

$$
\mu=\operatorname{dim}_{C} \mathcal{O}_{C^{n}, 0} /\left(\frac{\partial F}{\partial x_{1}}, \ldots, \frac{\partial F}{\partial x_{n}}\right) .
$$

These results were extended to the case $1<k<n$ by Hamm [Ha], who proved that the Milnor fiber has the homotopy type of a bouquet of $\mu(n-k)$-spheres, and by Lê [Le] and Greuel [Gr] who obtained the formula

$$
\mu\left(F^{\prime}\right)+\mu(F)=\operatorname{dim}_{C} \mathcal{O}_{C^{n}, 0} / I
$$

where $F^{\prime}=\left(f_{1}, \ldots, f_{k-1}\right)$ and $I$ is the ideal generated by $f_{1}, \ldots, f_{k-1}$ and all the $k \times k$ minors $\partial\left(f_{1}, \ldots, f_{k}\right) / \partial\left(x_{l_{1}}, \ldots, x_{l_{k}}\right)$.

For the real case, it is difficult to give such precise informations about the topology of the Milnor fiber. Nevertheless it is possible to compute some Euler characteristics. For instance, if $k=1$, the Khimshiasvili's formula ([Ar], $[\mathrm{Fu}]$, $[\mathrm{Kh}],[\mathrm{Wa}])$ states that

$$
\chi\left(F^{-1}(\delta) \cap B_{\varepsilon}\right)=1-\operatorname{sign}(-\delta)^{n} \operatorname{deg}_{0} \nabla F,
$$

where $\chi(-)$ denotes the Euler-Poincare characteristic and $\operatorname{deg}_{0} \nabla F$ is the topological degree of the gradient of $F$ at the origin.

It is also possible to compute the following difference

$$
D_{\delta, \alpha}=\chi\left(F^{-1}(\delta) \cap\{g \geq \alpha\} \cap B_{\varepsilon}\right)-\chi\left(F^{-1}(\delta) \cap\{g \leq \alpha\} \cap B_{\varepsilon}\right),
$$

for $(\delta, \alpha)$ a suitable regular value of $(F, g)$.

Recelved November 8, 1999; revised April 24, 2000. 
In [Dut2], we proved that

$$
D_{\delta, \alpha} \equiv \operatorname{dim}_{\boldsymbol{R}} \mathcal{O}_{\boldsymbol{R}^{n}, 0} / I_{g} \bmod 2
$$

where $I_{g}$ is the ideal generated by $f_{1}, \ldots, f_{k}$ and all $(k+1) \times(k+1)$ minors $\partial\left(g, f_{1}, \ldots, f_{k}\right) / \partial\left(x_{l_{1}}, \ldots, x_{i_{k+1}}\right)$, which generalized the case $g=x_{1}^{2}+\cdots+x_{n}^{2}$ already shown by Duzinski et al. [DLNS]. This is a general and effective formula, but it is only a mod 2 relation and one may ask when it is possible to get a more precise relation.

When $k=n-1$ and $g=x_{1}^{2}+\cdots+x_{n}^{2}$, according to Aoki et al. ([AFN1], [AFS]), $D_{\delta, 0}=\chi\left(F^{-1}(\delta) \cap B_{\varepsilon}\right)=\operatorname{deg}_{0} H$ and $2 \operatorname{deg}_{0} H$ is the number of halfbranches of $F^{-1}(0)$, where

$$
H=\left(\frac{\partial\left(g, f_{1}, \ldots, f_{n-1}\right)}{\partial\left(x_{1}, \ldots, x_{n}\right)}, f_{1} \ldots, f_{n-1}\right) .
$$

They generalized this result to the case $g=x_{n}$ in [AFN2] and Szafraniec generalized it to any $g$ is $[\mathrm{Szl} 1$. For other results concerning the numbers of halfbranches of a real curve, the reader may refer to [Da1], [Da2], [Da3], [MvS].

When $k=1$ and $g=x_{1}$, Fukui [Fu1] stated that $D_{\delta, 0}=-\operatorname{sign}(-\delta)^{n} \operatorname{deg}_{0} H$ where $H=\left(F, \partial F / \partial x_{2}, \ldots, \partial F / \partial x_{n}\right)$. We gave independently a similar result for any $g$ such that $\nabla g(0) \neq 0$ in [Dut1].

Fukui [Fu2] generalizes this formula when $F$ is a 2-parameter bifurcation of an $n$-dimensional function-germ, $g$ depends only on the two parameters and $(\delta, 0)$ is a regular value of $(F, g)$. In this paper, following Fukui's method, we will give a degree formula for $D_{\delta, 0}$ in a more general setting.

First we introduce the situation and some notations. For an analytic map $F: X \rightarrow \boldsymbol{R}^{p}$ on an analytic space $X$, we denote by $\Sigma(F)$ the singular locus of $F$ and by $Z(F)$ its zero locus.

Let $x=\left(x_{1}, \ldots, x_{p}\right)$ be a system of coordinates of $\boldsymbol{R}^{p}$ at the origin, $y=$ $\left(y_{1}, \ldots, y_{q}\right)$ a system of coordinates of $\boldsymbol{R}^{q}$ at the origin, and $z=\left(z_{1}, \ldots, z_{n}\right)$ a system of coordinates of $\boldsymbol{R}^{n}$ at the origin. Let $f:\left(\boldsymbol{R}^{p+q+n}, 0\right) \rightarrow(\boldsymbol{R}, 0)$ be a $(p+q)$-parameter bifurcation of an $n$-dimensional function-germ, $g:\left(\boldsymbol{R}^{p+q+n}, 0\right)$ $\rightarrow(\boldsymbol{R}, 0)$ an analytic function and $h=\left(h_{1}, \ldots, h_{p}\right):\left(\boldsymbol{R}^{p+q+n}, 0\right) \rightarrow\left(\boldsymbol{R}^{p}, 0\right)$ an analytic map. We write $g_{z}=\left(\partial g / \partial z_{1}, \ldots, \partial g / \partial z_{n}\right)$ and

$$
h_{z}=\left(\begin{array}{ccc}
\frac{\partial h_{1}}{\partial z_{1}} & \cdots & \frac{\partial h_{1}}{\partial z_{n}} \\
\vdots & & \vdots \\
\frac{\partial h_{p}}{\partial z_{1}} & \cdots & \frac{\partial h_{p}}{\partial z_{n}}
\end{array}\right) .
$$

We also use the similar notation, that is, $f_{x}, g_{x}, h_{x}, f_{y}, g_{y}, h_{y}, f_{z}, \ldots$ which mean the corresponding submatrices of the Jacobian matrices. 
We will use the following notations:

$$
\begin{aligned}
& F_{\delta}=f^{-1}(\delta) \cap h^{-1}(0) \cap B_{\varepsilon}, \\
& F_{\delta}(g \geq 0)=F_{\delta} \cap\{g \geq 0\}, \\
& F_{\delta}(g \leq 0)=F_{\delta} \cap\{g \leq 0\} .
\end{aligned}
$$

where $B_{\varepsilon}$ is a small ball centered at the origin and $\delta$ is close to 0 .

Now we consider the map

$$
\begin{aligned}
H=H(g, f, h):\left(\boldsymbol{R}^{p+q+n}, 0\right) & \rightarrow\left(\boldsymbol{R}^{p+q+n}, 0\right) \\
(x, y, z) & \mapsto\left(f, h, m, f_{z}\right),
\end{aligned}
$$

where $m$ is a map $\left(\boldsymbol{R}^{p+q+n}, 0\right) \rightarrow\left(\boldsymbol{R}^{q-1}, 0\right)$ defined by

$$
\begin{array}{rlrl}
m= & m_{12} & q=2, \\
m= & \left(m_{12}+m_{34}, m_{13}-m_{24}, m_{14}+m_{23}\right) & q=4, \\
m= & \left(m_{12}+m_{34}+m_{56}+m_{78}, m_{13}-m_{24}-m_{57}+m_{68},\right. & \\
& m_{14}+m_{23}+m_{58}+m_{67}, m_{15}-m_{26}+m_{37}-m_{48}, & \\
& m_{16}+m_{25}-m_{38}-m_{47}, m_{17}-m_{28}-m_{35}+m_{46}, & \\
& \left.m_{18}+m_{27}+m_{36}+m_{45}\right) & q=8,
\end{array}
$$

where $m_{i j}=\partial(g, h, f) / \partial\left(x, y_{i}, y_{j}\right)$.

We assume that $q=2,4,8$ and that $g$ and $h$ do not depend on $z$. The latter condition implies that $g_{z}$ and $h_{z}$ are identically zero. We also assume that

(A): $H$ is a finite map germ at 0 ,

(B): $(\delta, 0,0)$ is a regular value of $(f, g, h)$ when $0<|\delta| \ll 1$, and

(C): $Z\left(m, f_{z}, h\right) \cap \Sigma_{x} \subset\{0\}$ near 0 ,

where $\Sigma_{x}=\left\{s \in B_{\varepsilon}|| h_{x} \mid=0\right.$ at $\left.s\right\}$. When $p=0$, we understand $\Sigma_{x}=\emptyset$.

We prove (see Theorem 4.3):

$$
\operatorname{deg} H=(-1)^{p-1} \operatorname{sign}(-\delta)^{n}\left\{\chi\left(F_{\delta}(g \geq 0)\right)-\chi\left(F_{\delta}(g \leq 0)\right)\right\} .
$$

Then we describe the case $p=n=0$. We obtain the following formula (see Theorem 5.2):

$$
-2 \operatorname{deg} H=\chi\left(f^{-1}(0) \cap\{g \geq 0\} \cap S_{\varepsilon}\right)-\chi\left(f^{-1}(0) \cap\{g \leq 0\} \cap S_{\varepsilon}\right),
$$

where $S_{\varepsilon}$ is the sphere centered at the origin with radius $\varepsilon, f^{-1}(0) \cap S_{\varepsilon}$ is the link of $f:\left(\boldsymbol{R}^{q}, 0\right) \rightarrow(\boldsymbol{R}, 0)$ and $H:\left(\boldsymbol{R}^{q}, 0\right) \rightarrow\left(\boldsymbol{R}^{q}, 0\right), x \mapsto(f, m)$ with $m$ defined by (1) and $m_{i j}=\partial(g, f) / \partial\left(y_{i}, y_{j}\right)$.

It is proper to mention that recently Szafraniec [Sz3] has found a new effective method for computing topological invariants of real analytic singularities. He expresses

$$
\chi\left(F^{-1}(y) \cap B_{\varepsilon}\right) \text { and } D_{y, 0}
$$

as the signature of a matrix whose entries are analytic functions in $y$. 
The paper is organized as follows: in Section 2, we recall some facts about Morse theory for manifolds with boundary; in Section 3 and Section 4 are devoted to the proof of our main result; in Section 5 , we study the case $p=n=0$ and in Section 6, we give some examples.

The author thanks Karim Bekka and Zbigniew Szafraniec for their advices and for their interest in this paper. He is also very grateful to the referee for his helpful remarks and suggestions of improvement.

\section{Morse theory for manifolds with boundary}

We recall the results of Morse theory for manifolds with boundary. Our reference is $[\mathrm{HL}]$ where the results are given for a $C^{\infty}$ manifold $M$ with boundary $\partial M$. For simplicity we will present the results for manifolds with boundary of type $M \cap\{g * 0\}, * \in\{\geq, \leq\}$, where $M$ is a $C^{\infty}$ manifold and $g: M \rightarrow \boldsymbol{R}$ a $C^{\infty}$ function such that $M \cap g^{-1}(0)$ is smooth. In fact this is the case we need in the following sections.

Let $M$ be a $C^{\infty}$ manifold of dimension $n$. Let $g: M \rightarrow \boldsymbol{R}$ be a $C^{\infty}$ function such that $\nabla g(x) \neq 0$ for all $x \in g^{-1}(0)$. This implies that $M \cap g^{-1}(0)$ is a smooth manifold of dimension $n-1$ and that $M \cap\{g \geq 0\}$ and $M \cap\{g \leq 0\}$ are smooth manifolds with boundary. Let $f: M \rightarrow \boldsymbol{R}$ be a smooth function. A critical point of $f_{\mid M \cap\{g \geq 0\}}$ (resp. $f_{\mid M \cap\{g \leq 0\}}$ ) is a critical point of $f_{\mid M \cap\{g>0\}}$ (resp. $\left.f_{\mid M \cap\{g<0\}}\right)$ or a critical point of $f_{\mid M \cap g^{-1}(0)}$.

Definition 2.1. Let $q \in M \cap g^{-1}(0)$. We say that $q$ is a correct critical point of $f_{\mid M \cap\{g \geq 0\}}$ (resp. $f_{\mid M \cap\{g \leq 0\}}$ ) if $q$ is a critical point of $f_{\mid M \cap g^{-1}(0)}$ and $q$ is not a critical point of $f_{\mid M}$.

We say that $q$ is a correct non-degenerate critical point of $f_{\mid M \cap\{g \geq 0\}}$ (resp. $\left.f_{\mid M \cap\{g \leq 0\}}\right)$ if $q$ is a correct critical point of $f_{\mid M \cap\{g \geq 0\}}$ (resp. $f_{\mid M \cap\{g \leq 0\}}$ ) and $q$ is a non-degenerate critical point of $f_{\mid M \cap g^{-1}(0)}$.

If $q$ is a correct critical point of $f_{\mid M \cap\{g \geq 0\}}$ (resp. $f_{\mid M \cap\{g \leq 0\}}$ ) then $\nabla f(q) \neq \overrightarrow{0}$, $\nabla f(q)$ and $\nabla g(q)$ are colinear and there is $\tau(q) \in \boldsymbol{R}^{*}$ with $\nabla f(q)=\tau(q) \cdot \nabla g(q)$.

DEFINITION 2.2. If $q$ is a correct critical point of $f_{\mid M \cap\{g \geq 0\}}$ then

- $\nabla f(q)$ points inwards if and only if $\tau(q)>0$,

- $\nabla f(q)$ points outwards if and only if $\tau(q)<0$.

If $q$ is a correct critical point of $f_{\mid M \cap\{g \leq 0\}}$ then

- $\nabla f(q)$ points inwards if and only if $\tau(q)<0$,

- $\nabla f(q)$ points outwards if and only if $\tau(q)>0$.

Definition 2.3. A $C^{\infty}$ function $f: M \cap\{g \geq 0\} \rightarrow \boldsymbol{R}$ (resp. $M \cap\{g \leq 0\}$ $\rightarrow \boldsymbol{R})$ is a correct function if all critical points of $f_{\mid M \cap g^{-1}(0)}$ are correct. A $C^{\infty}$ function $f: M \cap\{g \geq 0\} \rightarrow \boldsymbol{R}$ (resp. $M \cap\{g \leq 0\} \rightarrow \boldsymbol{R}$ ) is a Morse correct function if $f_{\mid M \cap\{g>0\}}$ (resp. $\left.f_{\mid M \cap\{g<0\}}\right)$ admits only non-degenerate critical points and if $f$ admits only non-degenerate correct critical points. 
Proposition 2.4. For all $C^{\infty}$ manifold $M$ and for all function $g: M \rightarrow \boldsymbol{R}$ such that $\nabla g(x) \neq 0$ for all $x \in g^{-1}(0)$, the set of $C^{\infty}$ functions $f: M \rightarrow \boldsymbol{R}$ such that $f_{\mid M \cap\{g \geq 0\}}$ and $f_{\mid M \cap\{g \leq 0\}}$ are Morse correct functions is dense in $C^{\infty}(M, R)$.

We will denote $\chi(M \cap\{g * 0\} \cap\{f ? 0\})$, where $*, ? \in\{\leq,=, \geq\}$, by $\chi_{*, ?}$ and we will use the following result:

THEOREM 2.5. Let $M$ be a $C^{\infty}$ compact manifold of dimension $n$ and let $g$ : $M \rightarrow \boldsymbol{R}$ be a $C^{\infty}$ function such that $\nabla g(x) \neq 0$ for all $x \in g^{-1}(0)$. Let $f: M \rightarrow \boldsymbol{R}$ be a $C^{\infty}$ function such that $f_{\mid M \cap\{g \geq 0\}}$ and $f_{\mid M \cap\{g \leq 0\}}$ are Morse correct. Let $\left\{p_{i}\right\}$ be the set of critical points of $f_{\mid M}$ and $\left\{\lambda_{l}\right\}$ be the set of their respective indices. Let $\left\{q_{j}\right\}$ be the set of critical points of $f_{\mid M \cap g^{-1}(0)}$ and $\left\{\mu_{j}\right\}$ be the set of their respective indices. Then we have

$$
\begin{gathered}
\chi_{\geq, \geq}-\chi_{\geq,=}=\sum_{\substack{i / f\left(p_{i}\right)>0 \\
g\left(p_{i}\right)>0}}(-1)^{\lambda_{l}}+\sum_{\substack{J / f\left(q_{j}\right)>0 \\
\tau\left(q_{J}\right)>0}}(-1)^{\mu_{j}} \\
\chi_{\geq, \leq}-\chi_{\geq,=}=(-1)^{n} \sum_{\substack{i / f\left(p_{i}\right)<0 \\
g\left(p_{l}\right)>0}}(-1)^{\lambda_{l}}+(-1)^{n-1} \sum_{\substack{J / f\left(q_{j}\right)<0 \\
\tau\left(q_{j}\right)<0}}(-1)^{\mu_{j}},
\end{gathered}
$$

and

$$
\begin{gathered}
\chi_{\leq, \geq}-\chi_{\leq,=}=\sum_{\substack{l / f\left(p_{i}\right)>0 \\
g\left(p_{i}\right)<0}}(-1)^{\lambda_{l}}+\sum_{\substack{J / f\left(q_{j}\right)>0 \\
\tau\left(q_{J}\right)<0}}(-1)^{\mu_{j}}, \\
\chi_{\leq, \leq}-\chi_{\leq,=}=(-1)^{n} \sum_{\substack{u / f\left(p_{i}\right)<0 \\
g\left(p_{i}\right)<0}}(-1)^{\lambda_{l}}+(-1)^{n-1} \sum_{\substack{J / f\left(q_{j}\right)<0 \\
\tau\left(q_{j}\right)>0}}(-1)^{\mu_{J}} .
\end{gathered}
$$

\section{Preliminaries}

Before starting our work, we recall a lemma in linear algebra (for a proof, see $[\mathrm{Mu}])$.

Lemma (LAW of Extensible Minors). Let $A=\left(a_{i j}\right)_{i, j=1, \ldots, n}$ be a square matrix of order $n$. We denote by $A_{I J}$ the corresponding minors $\left|a_{i, j}\right|_{i \in I ; j \in J}$ of $A$ for $I, J \subset\{1, \ldots, n\}$ with $\sharp I=\sharp J$. We fix an integer $k, 1 \leq k \leq n$, and consider the submatrix $B=\left(a_{i j}\right)_{i, j=k, \ldots, n}$ of order $n-k+1$ of $A$. Assume that we have an identity between minors of $B$ which is a homogeneous polynomial in $a_{i j}$ for $i, j=k, \ldots, n$. Then the homogeneous formula obtained by replacing all the minors $A_{I J}$ of $B$ in the identity by $A_{\tilde{I} \tilde{I}}$ is also true, where $\tilde{I}=\{1, \ldots, k-1\} \cup I$, and $\tilde{J}=\{1, \ldots, k-1\} \cup J$. We remark that the 0 th minor 1 of $B$ in the identity is replaced by $A_{K, K}, K=\{1, \ldots, k-1\}$, so that the new formula becomes homogeneous. 
First we characterize $\Sigma\left(g_{\mid F_{\delta} \backslash Z(g)}\right)$. For subsets $X, Y$ and $Z$ of $B_{\varepsilon}$, we say $X=Y$ except $\mathrm{Z}$ if $X \backslash Z=Y \backslash Z$. We set

$$
\Sigma=\left\{s \in B_{\varepsilon} \mid \operatorname{rank}\left(\begin{array}{cc}
g_{x} & g_{y} \\
h_{x} & h_{y} \\
f_{x} & f_{y}
\end{array}\right)<p+2 \text { at } s\right\} .
$$

Lemma 3.1. $\Sigma\left(g_{\mid F_{\delta} \backslash Z(g)}\right)=F_{\delta} \cap \Sigma \cap Z\left(f_{z}\right)$ except $Z(g)$, when $0<|\delta| \ll \varepsilon \ll 1$.

Proof. Let $X$ denote the set $Z(h) \cap \Sigma(g, h) \backslash(\Sigma(h) \cup Z(g))$. Let $s \in \Sigma\left(g_{\mid F_{\delta} \backslash Z(g)}\right)$. This means that $s \in F_{\delta} \backslash Z(g)$ and

$$
\operatorname{rank}\left(\begin{array}{ccc}
g_{x} & g_{y} & 0 \\
h_{x} & h_{y} & 0 \\
f_{x} & f_{y} & f_{z}
\end{array}\right)<p+2 \text { at } s
$$

If there exits $k \in\{1, \ldots, n\}$ such that $f_{z_{k}}(s) \neq 0$, then $\operatorname{rank}\left(\begin{array}{ll}g_{x} & g_{y} \\ h_{\mathrm{r}} & h_{y}\end{array}\right)<p+1$ at $s$ and $s \in X$, since $(\delta, 0)$ is a regular value of $(f, h)$ by condition (B). So it is enough to show that $X \subset\{0\}$ for sufficiently small $B_{\varepsilon}$. If $X$ contains points nearby 0 , there exists an analytic curve $\gamma:[0, v[\rightarrow \bar{X}, \gamma(0)=0$. Since $\gamma(t)$ is in $Z(h)$ for $t \neq 0$, we have that $\left\langle\nabla h_{j}(\gamma(t)), \gamma^{\prime}(t)\right\rangle=0$ for $j=1, \ldots, p$. Because $\gamma(t) \in \Sigma(g, h) \backslash \Sigma(h)$ for $t \neq 0, \nabla g, \nabla h_{1}, \ldots, \nabla h_{p}$ are linearly dependent along $\gamma(t)$. Thus there exists $\alpha_{J}, j=1, \ldots, p$, so that $\nabla_{g}=\sum_{J=1}^{p} \alpha_{j} \nabla h_{j}$ along $\gamma(t)$. Then we obtain

$$
\frac{d}{d t}\left(g(\gamma(t))=\left\langle\nabla g(\gamma(t)), \gamma^{\prime}(t)\right\rangle=\sum_{j=1}^{p} \alpha_{j}(\gamma(t))\left\langle\nabla h_{j}(\gamma(t)), \gamma^{\prime}(t)\right\rangle=0,\right.
$$

and thus $\gamma(t) \in Z(g)$, which is a contradiction. This implies that $\Sigma\left(g_{\mid F_{\delta} \backslash Z_{(g)}}\right)$ is included in $F_{\delta} \cap \Sigma \cap Z\left(f_{z}\right)$. The inverse inclusion is obvious.

Lemma 3.2. $\Sigma=Z(\tilde{m})$ except $\Sigma_{x}$ where $\tilde{m}=\left(m_{i j}\right)_{1 \leq l, J \leq q}$.

Proof. It is clear that $\Sigma \subset Z(\tilde{m})$. Now let $s \in Z(\tilde{m}) \backslash \Sigma_{x}$. Then $\operatorname{rank}\left(h_{x}\right)$ $=p$ at $s$. If there exists $i \in\{1, \ldots, q\}$ such that $\operatorname{rank}\left(\begin{array}{ll}g_{\mathrm{x}} & g_{y_{l}} \\ h_{\mathrm{r}} & h_{y_{l}} \\ f_{x} & f_{y_{l}}\end{array}\right)=p+1$ at $s$, then since for all $j \neq i, m_{i j}=0$ at $s, \quad \operatorname{rank}\left(\begin{array}{ll}g_{x} & g_{y} \\ h_{x} & h_{y} \\ f_{x} & f_{y}\end{array}\right)=p+1 \quad$ at $\quad s \quad$ and 
$s \in \Sigma$. If for all $i \in\{1, \ldots, q\}, \operatorname{rank}\left(\begin{array}{ll}g_{x} & g_{y_{l}} \\ h_{x} & h_{y_{t}} \\ f_{x} & f_{y_{l}}\end{array}\right)=p$ at $s$, then it is clear that $\operatorname{rank}\left(\begin{array}{ll}g_{x} & g_{y} \\ h_{x} & h_{y} \\ f_{x} & f_{y}\end{array}\right)=p$ at $s$ and $s \in \Sigma$. Hence $Z(\tilde{m})=\Sigma$ except $\Sigma_{x}$.

LeMma 3.3. $F_{\delta} \cap Z\left(\tilde{m}, f_{z}\right)=F_{\delta} \cap Z\left(m, f_{z}\right)$ except $\Sigma_{x}$.

Proof. The case $q=2$ is trivial. It is clear that $Z(\tilde{m}) \subset Z(m)$. Now let $s \in\left(F_{\delta} \cap Z\left(m, f_{z}\right)\right) \backslash \Sigma_{x}$. Condition (B) implies that $(\delta, 0)$ is a regular value of $(f, h)$, that is $\operatorname{rank}\left(\begin{array}{ccc}h_{x} & h_{y} & 0 \\ f_{x} & f_{y} & f_{z}\end{array}\right)=p+1$ at $s$ and so, $\operatorname{rank}\left(\begin{array}{cc}h_{x} & h_{y} \\ f_{x} & f_{y}\end{array}\right)=p+1$ at $s$ for $f_{z}$ vanish at $s$. Since $s \notin \Sigma_{x}, \operatorname{rank}\left(\begin{array}{c}h_{x} \\ f_{x}\end{array}\right)=p$ at $s$ and there exists $i \in\{1, \ldots, q\}$ with $\operatorname{rank}\left(\begin{array}{cc}h_{x} & h_{y_{1}} \\ f_{\mathrm{x}} & f_{y_{i}}\end{array}\right)=p+1$ at $s$. We can assume that $i=1$. If we write $f_{l}=\left|\begin{array}{ll}h_{x} & h_{y_{l}} \\ f_{x} & f_{y_{l}}\end{array}\right|$, we obtain that:

$$
m_{i j}=\left(f_{j} m_{1_{l}}-f_{i} m_{1 j}\right) / f_{1} \text { at } s, \quad 1 \leq i<j \leq q .
$$

When $p=0$, this comes from the expansion along the first row of $\left|\begin{array}{lll}f_{y_{1}} & f_{y_{1}} & f_{y_{j}} \\ g_{y_{1}} & g_{y_{i}} & g_{y_{j}} \\ f_{y_{1}} & f_{y_{i}} & f_{y_{j}}\end{array}\right|$. Applying the Law of Extensible Minors, we obtain the formula

(2) for general $p$.

We first consider the case $q=4$. Because of (2), we have

$$
\left(\begin{array}{l}
m_{12}+m_{23} \\
m_{13}-m_{24} \\
m_{14}+m_{23}
\end{array}\right)=A\left(\begin{array}{l}
m_{12} \\
m_{13} \\
m_{14}
\end{array}\right) \text { at } s, \quad \text { where } A=\frac{1}{f_{1}}\left(\begin{array}{ccc}
f_{1} & -f_{4} & f_{3} \\
f_{4} & f_{1} & -f_{2} \\
-f_{3} & f_{2} & f_{1}
\end{array}\right) .
$$

Since $|A|=\left(f_{1}^{2}+f_{2}^{2}+f_{3}^{2}+f_{4}^{2}\right) / f_{1}^{2}>0$ at $s, m_{12}=m_{13}=m_{14}=0$ at $s$ and, by (2), $m_{i j}=0$ at $s$ for $1 \leq i<j \leq q$, which means that $s \in Z(\tilde{m})$.

When $q=8$, similar computations shows

$$
A=\frac{1}{f_{1}}\left(\begin{array}{ccccccc}
f_{1} & -f_{4} & f_{3} & -f_{6} & f_{5} & -f_{8} & f_{7} \\
f_{4} & f_{1} & -f_{2} & f_{7} & -f_{8} & -f_{5} & f_{6} \\
-f_{3} & f_{2} & f_{1} & -f_{8} & -f_{7} & f_{6} & f_{5} \\
f_{6} & -f_{7} & f_{8} & f_{1} & -f_{2} & f_{3} & -f_{4} \\
-f_{5} & f_{8} & f_{7} & f_{2} & f_{1} & -f_{4} & -f_{3} \\
f_{8} & f_{5} & -f_{6} & -f_{3} & f_{4} & f_{1} & -f_{2} \\
-f_{7} & -f_{6} & -f_{5} & f_{4} & f_{3} & f_{2} & f_{1}
\end{array}\right)
$$


and we have to show that $|A|>0$ at $s$. Actually, we will prove that $|A|=$ $\left(\sum_{l=1}^{8} f_{l}^{2}\right)^{3} / f_{1}^{6}$. Let us consider the following matrices

$$
A(u)=\frac{1}{f_{1}}\left(\begin{array}{ccccccc}
f_{1} & -f_{4} \cdot u & f_{3} \cdot u & -f_{6} \cdot u & f_{5} \cdot u & -f_{8} \cdot u & f_{7} \cdot u \\
f_{4} \cdot u & f_{1} & -f_{2} \cdot u & f_{7} \cdot u & -f_{8} \cdot u & -f_{5} \cdot u & f_{6} \cdot u \\
-f_{3} \cdot u & f_{2} \cdot u & f_{1} & -f_{8} \cdot u & -f_{7} \cdot u & f_{6} \cdot u & f_{5} \cdot u \\
f_{6} \cdot u & -f_{7} \cdot u & f_{8} \cdot u & f_{1} & -f_{2} \cdot u & f_{3} \cdot u & -f_{4} \cdot u \\
-f_{5} \cdot u & f_{8} \cdot u & f_{7} \cdot u & f_{2} \cdot u & f_{1} & -f_{4} \cdot u & -f_{3} \cdot u \\
f_{8} \cdot u & f_{5} \cdot u & -f_{6} \cdot u & -f_{3} \cdot u & f_{4} \cdot u & f_{1} & -f_{2} \cdot u \\
-f_{7} \cdot u & -f_{6} \cdot u & -f_{5} \cdot u & f_{4} \cdot u & f_{3} \cdot u & f_{2} \cdot u & f_{1}
\end{array}\right)
$$

and let $P: \boldsymbol{R} \rightarrow \boldsymbol{R}$ be defined by $P(u)=|A(u)|$ for $u \in \boldsymbol{R}$. It is clear that $P$ is a polynomial function in $u$ and that $P(1)=|A|$ and $P(0)=1$. Now let us compute $P(u)$ for any $u$. Setting

$$
B(u)=\left(\begin{array}{cc}
1 & f_{l} \cdot u / f_{1} \\
0 & A(u)
\end{array}\right)
$$

we obtain

$$
{ }^{t} B(u) B(u)=\left(\begin{array}{cc}
1 & f_{l} \cdot u / f_{1} \\
f_{l} \cdot u / f_{1} & \phi(u) I_{7}
\end{array}\right),
$$

where $\phi(u)=1+\left(\sum_{l=2}^{8}\left(f_{l} \cdot u\right)^{2}\right) / f_{1}^{2}$ and $I_{7}$ is the unit matrix of order 7. This equality comes from the fact that ${ }^{t} B(u) B(u)=\left(\left\langle L_{i}(u), L_{j}(u)\right\rangle\right)$ where $L_{i}(u)$ denotes the $i^{\text {th }}$ column of $B(u)$ and $\langle$,$\rangle is the usual scalar product in \boldsymbol{R}^{8}$. Then we have

$$
P(u)^{2}=|A(u)|^{2}=\left|\begin{array}{cc}
1 & f_{l} \cdot u / f_{1} \\
f_{l} \cdot u / f_{1} & \phi(u) I_{7}
\end{array}\right|,
$$

and, expanding this determinant along the first row and the first column, we obtain $P(u)^{2}=\phi(u)^{6}$, which implies that $P$ has no real root and so keeps a constant sign. Since $P(0)=1$, we can conclude that $|A|=\phi(1)^{3}=\left(\sum_{l=1}^{8} f_{l}^{2}\right)^{3} / f_{1}^{6}$.

LEMMA 3.4. $\Sigma\left(g_{\mid F_{\delta} \backslash Z_{(g)}}\right)=\left(F_{\delta} \cap Z\left(m, f_{z}\right)\right) \backslash Z(g)$.

Proof. By the three previous lemmas, we have

$$
\Sigma\left(g_{\mid F_{\delta} \backslash Z_{(g)}}\right)=F_{\delta} \cap Z\left(m, f_{z}\right) \text { except } \Sigma_{x} \cup Z(g) .
$$

But condition (C) implies that $Z\left(m, f_{z}, h\right) \cap \Sigma_{x} \cap f^{-1}(\delta)=\emptyset$ when $0<|\delta| \ll 1$.

Keeping the notations introduced in the proof of Lemma 3.3, we write $g_{j}=\left|\begin{array}{ll}h_{\mathrm{r}} & h_{y_{j}} \\ g_{x} & g_{y_{j}}\end{array}\right|$ for $1 \leq j \leq q$. 
Lemma 3.5. Let $s \in \Sigma\left(g_{\mid F_{\delta} \backslash Z_{(g)}}\right)$. Then $f_{1}(s) \neq 0$ implies that $g_{1}(s) \neq 0$.

Proof. By condition (B), $s \in Z(h) \backslash \Sigma(h)$ and then $s \notin Z(g, h)$ otherwise $g$ would vanish at $s$, as proved in Lemma 3.1. This implies that there exists $i \in$ $\{1, \ldots, q\}$ with $g_{i}(s) \neq 0$. Now we see the following

$$
\left|\begin{array}{cc}
g_{1} & g_{i} \\
f_{1} & f_{l}
\end{array}\right|=(-1)^{p}\left|h_{x}\right| m_{1 l} \text { at } s .
$$

This is obvious when $p=0$. The case $p>0$ is obtain by applying the Law of Extensible Minors to the case $p=0$. Since $s \in \Sigma\left(g_{\left|F_{\delta}\right| Z_{(q)}}\right)$, we have that $m_{1 i}(s)=0$. If $g_{1}(s)=0$ then $f_{1}(s) \times g_{i}(s)=0$ and $f_{1}(s)=0$.

From now on, we assume that $s \in \Sigma\left(g_{\mid F_{\delta} \backslash Z_{(g)}}\right)$ and $f_{1}(s) \neq 0$. We set $y^{\prime}=$ $\left(y_{2}, \ldots, y_{q}\right)$. By the Implicit Function Theorem, there exists a map

$$
\varphi: \boldsymbol{R}^{p+n-1} \rightarrow \boldsymbol{R}^{p} \times \boldsymbol{R},\left(y^{\prime}, z\right) \mapsto\left(\varphi_{x}\left(y^{\prime}, z\right), \varphi_{1}\left(y^{\prime}, z\right)\right),
$$

defined by some domain so that the image of the map $\left(y^{\prime}, z\right) \mapsto\left(\varphi_{x}\left(y^{\prime}, z\right)\right.$, $\left.\varphi_{1}\left(y^{\prime}, z\right), y^{\prime}, z\right)$ covers a neighborhood of $s$ in $F_{\delta}$. We denote by $J$ the Jacobian of $H$, and set

$$
G\left(y^{\prime}, z\right)=g\left(\varphi_{x}\left(y^{\prime}, z\right), \varphi_{1}\left(y^{\prime}, z\right), y^{\prime}, z\right) .
$$

LEMma 3.6. The function $g_{\mid F_{\delta}}$ has a Morse critical point at $s$ if and only if $H(s)=(0, \delta, 0)$ and $J(s) \neq 0 . \quad$ In that case

$$
\operatorname{sign} \operatorname{Hess}(G)=(-1)^{p-1} \operatorname{sign}(J) \times \operatorname{sign}\left(\frac{-g_{1}}{f_{1}}\right)^{n} \text { at } s .
$$

Proof. Let $\bar{m}=\left(m_{12}, \ldots, m_{1 q}\right), \bar{H}=\left(h, f, \bar{m}, f_{z}\right)$ and $\bar{J}$ be its Jacobian. For $k \in\{1, \ldots, n\}$, let $\mu_{k}=\left|\begin{array}{ccc}g_{x} & g_{y_{1}} & 0 \\ h_{x} & h_{y_{1}} & 0 \\ f_{x} & f_{y_{1}} & f_{\mu_{k}}\end{array}\right|$. Let $\mu=\left(\mu_{1}, \ldots, \mu_{n}\right)$ and

$$
B=\left(\begin{array}{ccc}
h_{x} & h_{y} & h_{z} \\
f_{x} & f_{y} & f_{z} \\
\bar{m}_{x} & \bar{m}_{y} & \bar{m}_{z} \\
\mu_{x} & \mu_{y} & \mu_{z}
\end{array}\right) .
$$

As Szafraniec does in [Sz2], we obtain

hence

$$
\operatorname{Hess}(G)=\frac{(-1)^{(p+1)(p+q+n-p-1)}}{f_{1}^{p+q+n-(p+1)+1}}|B| \text { at } s,
$$

$$
\operatorname{Hess}(G)=\frac{(-1)^{(p+1)(q+n-1)}}{f_{1}^{q+n}}|B| \text { at } s \text {. }
$$


For $k \in\{1, \ldots, n\}, \quad \mu_{k}=f_{z_{k}}\left|\begin{array}{ll}g_{\mathrm{r}} & g_{\nu_{1}} \\ h_{\mathrm{r}} & h_{y_{1}}\end{array}\right|=(-1)^{p} f_{z_{k}} \cdot g_{1}$. By differentiating this equality and using the fact that $f_{z_{k}}(s)=0$, we obtain that

$$
|B|=(-1)^{p \times n} \times g_{1}^{n} \times \bar{J} \text { at } s,
$$

which gives

$$
\operatorname{Hess}(G)=(-1)^{p-1} \frac{\left(-g_{1}\right)^{n}}{f_{1}^{q+n}} \bar{J} \text { at } s .
$$

Since $q$ is even, it remains to prove that $\operatorname{sign}(J)=\operatorname{sign}(\bar{J})$ at $s$. When $q=2$, this is trivial. For the other cases, we consider the matrix $A$ so that ${ }^{t} m=A^{t} \bar{m}$. Then we have

$$
H=\left(\begin{array}{lll}
1 & 0 & 0 \\
0 & A & 0 \\
0 & 0 & 1
\end{array}\right) \bar{H}
$$

Differentiating this equality and using the fact $\bar{m}(s)=0$, we obtain $J=|A| \bar{J}$. As we mentionned in the proof of Lemma 3.3, $|A|$ is strictly positive at $s$ which concludes the proof.

LEMMA 3.7. If $f_{1} \neq 0$ at $s$ then $\operatorname{sign}(g / f)=\operatorname{sign}\left(g_{1} / f_{1}\right)$ at $s$.

Proof. We assume that there exists an analytic curve $\gamma:[0,1] \rightarrow Z\left(h, f_{z}\right)$ $\cap \Sigma, t \mapsto \gamma(t)$ with $\gamma(0)=0$ and $\gamma(1)=s$. Since $f_{1}(s) \neq 0$ and $g_{1}(s) \neq 0$, we can assume that $f_{1}$ and $g_{1}$ do not vanish along $\gamma(t), t \neq 0$. Since $h_{j}(\gamma(t)) \equiv 0, j=$ $1, \ldots, p$ we obtain that

$$
0=h_{J}^{\prime}=\sum_{l=1}^{p} \frac{\partial h_{j}}{\partial x_{l}} \cdot \xi_{l}+\sum_{k=1}^{q} \frac{\partial h_{j}}{\partial y_{k}} \cdot \eta_{k} \text { along } \gamma(t), \quad j=1, \ldots, p
$$

where ' denotes the derivative by $t, \xi_{l}=\left(x_{l} \circ \gamma\right)^{\prime}, i=1, \ldots, p, \eta_{k}=\left(y_{k} \circ \gamma\right)^{\prime}$, $k=1, \ldots, q$. We also have

$$
f^{\prime}=\sum_{l=1}^{p} \frac{\partial f}{\partial x_{l}} \cdot \xi_{l}+\sum_{k=1}^{q} \frac{\partial f}{\partial y_{k}} \cdot \eta_{k} \text { along } \gamma(t) .
$$

Then, solving (3) and (4) by Cramer's rule, we obtain

$$
\begin{gathered}
\eta_{1}=\frac{1}{f_{1}}\left(\left|h_{x}\right| f^{\prime}-\sum_{k=2}^{q} f_{k} \cdot \eta_{k}\right), \\
\xi_{l}=\frac{(-1)^{p+l-1}}{f_{1}}\left(\left|h_{\hat{x}_{t}} h_{y_{1}}\right| f^{\prime}-\sum_{k=2}^{q}\left|\begin{array}{lll}
h_{\hat{x}_{i}} & h_{y_{1}} & h_{y_{k}} \\
f_{\hat{x}_{t}} & f_{y_{1}} & f_{y_{k}}
\end{array}\right| \eta_{k}\right),
\end{gathered}
$$

where $\hat{x}_{l}=\left(x_{1}, \ldots, x_{l-1}, x_{l+1}, \ldots, x_{p}\right)$. Then we obtain that 


$$
g^{\prime}=\sum_{l=1}^{p} \frac{\partial g}{\partial x_{l}} \cdot \xi_{l}+\sum_{k=1}^{q} \frac{\partial g}{\partial y_{k}} \cdot \eta_{k}=\frac{g_{1}}{f_{1}} f^{\prime}+(-1)^{p+1} \frac{1}{f_{1}} \sum_{k=2}^{q} m_{1 k} \cdot \eta_{k}
$$

so

$$
g^{\prime}=\frac{g_{1}}{f_{1}} f^{\prime} \text { along } \gamma(t)
$$

This implies the lemma.

LEMMA 3.8. Assume that $s$ is a non-degenerate critical point of $g_{\mid F_{\delta}}$ such that $g(s) \neq 0$. Then

1. if $g(s)>0$ then the function $g_{\mid F_{\delta}}$ has even (resp. odd) index at $s$ if and only if $H$ has local degree $(-1)^{p-1} \operatorname{sign}(-\delta)^{n}\left(\right.$ resp. $\left.(-1)^{p} \operatorname{sign}(-\delta)^{n}\right)$ at $s$,

2. if $g(s)<0$ then the function $g_{\mid F_{\delta}}$ has even (resp. odd) index at $s$ if and only if $H$ has local degree $(-1)^{p-1} \operatorname{sign} \delta^{n}\left(\right.$ resp. $\left.(-1)^{p} \operatorname{sign} \delta^{n}\right)$ at $s$.

Proof. The proof is just a combination of the two previous lemmas.

\section{Main result}

We will use Morse theory for manifolds with boundary. We need the two following lemmas.

Lemma 4.1. Assume that $(\delta, 0)$ is a regular value of $(f, h)$ for $0<|\delta| \ll$ $\varepsilon \ll 1$. Then

- All correct critical points of $g_{\mid F_{\delta}}$ with $g>0$ point outwards.

- All correct critical points of $g_{\mid F_{\delta}}$ with $g<0$ point inwards.

- There are no correct critical points of $g_{\mid F_{\delta}}$ with $g=0$.

Proof. We prove the first point, the second will follow considering the function $-g$. Recall that $F_{0}=Z(f, h) \cap B_{\varepsilon}$. Let $\omega$ be the Euclidian distance function and let

$$
\begin{gathered}
X=\left\{x \in F_{0} \backslash\{0\} \cap\{g(x)>0\} \mid \text { there exist } \lambda, \lambda_{1}, \ldots, \lambda_{p} \text { and } \mu\right. \\
\text { with } \left.\nabla g=\lambda \nabla f+\sum_{l=1}^{p} \lambda_{i} \nabla h_{i}+\mu \nabla \omega \text { and } \mu<0\right\} .
\end{gathered}
$$

It is a subanalytic set. If $0 \in \bar{X}$, we apply the curve selection lemma (cf [Mi2]). There exists $\gamma:\left[0, \varepsilon_{0}\left[\rightarrow \bar{X}\right.\right.$ analytic such that $\gamma(0)=0$. Then we have $(g \circ \gamma)^{\prime}=$ $\left\langle\nabla g(\gamma), \gamma^{\prime}\right\rangle$ and

$$
(g \circ \gamma)^{\prime}=\lambda\left\langle\nabla f(\gamma), \gamma^{\prime}\right\rangle+\sum_{i=1}^{p} \lambda_{i}\left\langle\nabla h_{i}(\gamma), \gamma^{\prime}\right\rangle+\mu\left\langle\nabla \omega(\gamma), \gamma^{\prime}\right\rangle .
$$


We have $\left\langle\nabla f(\gamma), \gamma^{\prime}\right\rangle=0$ for $f \circ \gamma \equiv 0$. Similarly for $i \in\{1, \ldots, p\},\left\langle\nabla h_{i}(\gamma), \gamma^{\prime}\right\rangle$ $=0$. Since $\left\langle\nabla \omega(\gamma), \gamma^{\prime}\right\rangle \geq 0$ for $t \in\left[0, \varepsilon_{0}[\right.$, we will have

$$
g \circ \gamma^{\prime} \leq 0 \text { for } t \in\left[0, \varepsilon_{0}[\text {. }\right.
$$

The function $g \circ \gamma$ is decreasing and so, for all $t \in\left[0, \varepsilon_{0}[, g \circ \gamma(t) \leq g \circ \gamma(0)=0\right.$. But $g>0$ along $\gamma(t)$ so $0 \notin \bar{X}$. We can choose $\varepsilon$ sufficiently small such that correct critical points of $g_{\mid F_{0} \backslash\{0\}}$ point outwards. Choosing $\delta$ sufficiently close to 0 , correct critical points of $g_{\mid F_{\delta}}$ will also point outwards.

We prove the third point with the same ideas considering the sets

$$
\begin{gathered}
Y_{>}=\left\{x \in F_{0} \backslash\{0\} \cap Z(g) \mid \text { there exist } \lambda, \lambda_{1}, \ldots, \lambda_{p} \text { and } \mu\right. \\
\text { with } \left.\nabla g=\lambda \nabla f+\sum_{l=1}^{p} \lambda_{l} \nabla h_{i}+\mu \nabla \omega \text { and } \mu>0\right\},
\end{gathered}
$$

and

$$
\begin{gathered}
Y_{<}=\left\{x \in F_{0} \backslash\{0\} \cap Z(g) \mid \text { there exist } \lambda, \lambda_{1}, \ldots, \lambda_{p} \text { and } \mu\right. \\
\text { with } \left.\nabla g=\lambda \nabla f+\sum_{l=1}^{p} \lambda_{l} \nabla h_{i}+\mu \nabla \omega \text { and } \mu<0\right\},
\end{gathered}
$$

and proving that $0 \notin \overline{Y_{>}}$and that $0 \notin \overline{Y_{<}}$.

Lemma 4.2. If there exists $\delta$ such that $(\delta, 0)$ is a regular value of $(f, h)$ then we can choose $\delta$ small enough and we can perturbe $f$ into $\tilde{f}$ in such a way that $g_{\mid \tilde{F}_{\delta}}$ has only Morse critical points in $\tilde{F}_{\delta} \backslash g^{-1}(0)$, where $\tilde{F}_{\delta}$ denotes $\tilde{f}^{-1}(\delta) \cap Z(h) \cap B_{\varepsilon}$.

Proof. We give the proof for $p=0$ and $q=2$. The proof of the general case is just an adaptation. Let

$$
\left(y_{1}, y_{2} ; z_{1}, \ldots, z_{n} ; u_{1}, u_{2} ; t_{1}, \ldots, t_{n}\right)=(y ; z ; u ; t)
$$

be a coordinate system of $\boldsymbol{R}^{2+n} \times \boldsymbol{R}^{2+n}$ and let

$$
\begin{gathered}
F(y, z, u, t)=f(y, z)+u_{1} y_{1}+u_{2} y_{2}+t_{1} z_{1}+\cdots+t_{n} z_{n} \\
G(y, z, u, t)=g(y, z) .
\end{gathered}
$$

We have for $i \in\{1, \ldots, n), F_{z_{l}}=f_{z_{l}}+t_{l}$. We also have

$$
\frac{\partial(G, F)}{\partial\left(y_{1}, y_{2}\right)}=G_{y_{1}}\left(f_{y_{2}}+u_{2}\right)-G_{y_{2}}\left(f_{y_{1}}+u_{1}\right) \text {. }
$$

Write $M_{12}=\partial(G, F) / \partial\left(y_{1}, y_{2}\right)$ and $X=Z\left(M_{12}, F_{z_{1}}, \ldots, F_{z_{n}}\right)$. Let 


$$
\Gamma=\left(\begin{array}{cc}
M_{12 y} & M_{12 z} \\
F_{z_{1 y}} & F_{z_{1 z}} \\
\vdots & \vdots \\
F_{z_{n y}} & F_{z_{n z}}
\end{array}\right) .
$$

It is the Jacobian matrix of $\left(M_{12}, F_{z_{1}}, \ldots, F_{z_{n}}\right): \boldsymbol{R}^{2+n} \times \boldsymbol{R}^{2+n} \rightarrow \boldsymbol{R}^{n+1}$. It is a $(n+2) \times 2(n+2)$ matrix which has the following form

$$
\Gamma(y, z, u, t)=\left(\begin{array}{cccccccc}
* & \cdots & * & G_{y_{1}} & G_{y_{2}} & 0 & \cdots & 0 \\
* & \cdots & * & 0 & 0 & 1 & \cdots & 0 \\
\vdots & \ddots & \vdots & \vdots & \vdots & & \ddots & \vdots \\
* & \cdots & * & 0 & 0 & 0 & \cdots & 1
\end{array}\right) .
$$

Now let

$$
\begin{aligned}
\pi: \boldsymbol{R}^{2+n} \times \boldsymbol{R}^{2+n} & \rightarrow \boldsymbol{R}^{3+n} \\
(y, z, u, t) & \mapsto(F(y, z, u, t), u, t),
\end{aligned}
$$

and let us call $\tilde{\pi}$ its restriction to $X$.

If $X$ is included in $G^{-1}(0)$ then for all $(\delta, u, t) \in \boldsymbol{R}^{n+3}, \tilde{\pi}^{-1}(\delta, u, t) \subset G^{-1}(0)$ and this means that for all $(\delta, u, t) \in \boldsymbol{R}^{n+3}, g_{\mid f_{u, t}^{-1}(\delta)}$, where $f_{u, t}(y, z)=F(y, z, u, t)$, has no critical point in $\{g \neq 0\}$ so $g_{\mid f_{u, t}^{-1}(\delta)}$ is a Morse function in $\{g \neq 0\}$.

If $X$ is not included in $G^{-1}(0)$ then $X \backslash G^{-1}(0)$ is a manifold of dimension $2(2+n)-(n+1)=n+3$ because for all $(y, z, u, t) \in X \backslash G^{-1}(0), G_{y_{1}}(y, z, u, t) \neq 0$ or $G_{y_{2}}(y, z, u, t) \neq 0$ and then $\operatorname{rank} \Gamma(y, z, u, t)=n+1$, so $X \backslash G^{-1}(0)$ is included in the set of regular points of $X$. By Bertini-Sard theorem we can choose $(\delta, u, t)$ near 0 in $\boldsymbol{R}^{n+3}$ such that $\pi$ is regular at each point in $\pi^{-1}(\delta, u, t) \cap\left(X \backslash G^{-1}(0)\right)$. This means that $g_{\mid f_{u t}^{-1}(\delta)}$ is a Morse function in $\{g \neq 0\}$.

THEOREM 4.3. Suppose that $f, h, g$ satisfy conditions (A), (B) and (C). Then

$$
\operatorname{deg} H=(-1)^{p-1} \operatorname{sign}(-\delta)^{n}\left\{\chi\left(F_{\delta}(g \geq 0)\right)-\chi\left(F_{\delta}(g \leq 0)\right)\right\} .
$$

Proof. Thanks to condition (A), $g_{\mid F_{0} \backslash\{0\}}$ has no critical point and then choosing $\delta$ sufficiently small, we can suppose that $g_{\mid f^{-1}(\delta) \cap Z(h) \cap\{\omega<\varepsilon\}}$ admits its critical points in $F_{\delta} \cap B_{\varepsilon / 4}$ because transversality is an open property. Thus the critical points of $g_{\mid f^{-1}(\delta) \cap Z(h) \cap\{\omega=\varepsilon\}}$ are correct critical points. By the two previous lemmas, we can assume that $g_{\mid F_{\delta}}$ is a correct Morse function, that its critical points are lying within $B_{\varepsilon / 2}$, that the correct critical points of $g_{\mid F_{\delta}}$ where $g>0$ (resp. $g<0$ ) point outwards (resp. inwards) and that there are no correct critical points of $g_{\mid F_{\delta}}$ where $g=0$. We apply Theorem 2.5 to the manifold with boundary $F_{\delta}(g \geq 0)$ and we get

$$
\chi\left(F_{\delta}(g \geq 0), F_{\delta}(g=0)\right)=n_{+}\left(g_{+}\right)-n_{-}\left(g_{+}\right),
$$


where $n_{+}\left(g_{+}\right)$(resp. $\left.n_{-}\left(g_{+}\right)\right)$is the number of non-degenerate critical points with even (resp. odd) index of $g_{\mid F_{\delta}(g \geq 0)}$. In the same way, we have

$$
\chi\left(F_{\delta}(g \leq 0), F_{\delta}(g=0)\right)=(-1)^{n+q-1}\left(n_{+}\left(g_{-}\right)-n_{-}\left(g_{-}\right)\right),
$$

where $n_{+}\left(g_{-}\right)$(resp. $\left.n_{-}\left(g_{-}\right)\right)$is the number of non-degenerate critical points with even (resp. odd) index of $g_{\mid F_{\delta}(g \leq 0)}$.

We have

$$
\operatorname{deg} H=\sum_{s \in H^{-1}(\varepsilon)} \operatorname{sign} J(s)
$$

where $\varepsilon$ is a regular value of $H$.

By Lemma 3.6, $s$ is a critical point of $g_{\mid F_{\delta}}$ if and only if $H(s)=(0, \delta, 0)$. Hence $H^{-1}\{(0, \delta, 0)\}$ is the set of critical points of $g_{\mid F_{\delta}}$. Since $g_{\mid F_{\delta}}$ is a Morse function, its critical points are non-degenerate and by Lemma 3.6, $J(s) \neq 0$ for $s \in H^{-1}\{(0, \delta, 0)\}$. We conclude that $(0, \delta, 0)$ is a regular value of $H$ and

$$
\operatorname{deg} H=\sum_{s \in H^{-1}(0, \delta, 0)} \operatorname{sign} J(s) .
$$

Conbining this with the above equalities and Lemma 3.7, we obtain the formula.

Now, if we replace condition $(\mathrm{B})$ by the weaker following condition:

$\left(\mathbf{B}^{\prime}\right):(\delta, 0)=(\delta, 0)$ is a regular value of $(f, h)$ when $0<|\delta| \ll 1$, we obtain Then

THEOREM 4.4. Suppose that $f, h, g$ satisfy conditions (A), (B') and (C).

$$
\operatorname{deg} H=(-1)^{p-1} \operatorname{sign}(-\delta)^{n}\left\{\chi\left(F_{\delta}(g \geq 0)\right)-\chi\left(F_{\delta}(g \leq 0)\right)\right\}+\mu_{(\delta, 0)},
$$

with $\mu_{(\delta, 0)}=\sum_{l} \operatorname{deg}_{q_{t}} H(\tilde{g}, f, h)$ where $\tilde{g}$ is a suitable perturbation of $g$ such that $\tilde{g}_{\mid F_{\delta}}$ is a Morse function and where the $q_{i}$ are the non-degenerate critical points of $\tilde{g}_{\mid F_{\delta}}$ lying near $F_{\delta}(g=0)$.

Proof. Choose $\alpha>0$ close to 0 such that 0 is the only critical value of $g_{\mid F_{\delta}}$ in $[-\alpha, \alpha]$. Then

$$
\chi\left(F_{\delta}(g \geq \alpha), F_{\delta}(g=\alpha)\right)=\chi\left(F_{\delta}(g \geq 0), F_{\delta}(g=0)\right),
$$

and

$$
\chi\left(F_{\delta}(g \leq-\alpha), F_{\delta}(g=-\alpha)\right)=\chi\left(F_{\delta}(g \leq 0), F_{\delta}(g=0)\right) .
$$

Replacing $f$ by a suitable perturbation if necessary we obtain

$$
\chi\left(F_{\delta}(g \geq 0), F_{\delta}(g=0)\right)=n_{+}\left(g_{\alpha}\right)-n_{-}\left(g_{\alpha}\right),
$$

where $n_{+}\left(g_{\alpha}\right)$ (resp. $n_{-}\left(g_{\alpha}\right)$ ) is the number of non-degenerate critical points with even (resp. odd) index of $g_{\mid F_{\delta}(g \geq \alpha)}$. Similarly, we have

$$
\chi\left(F_{\delta}(g \leq 0), F_{\delta}(g=0)\right)=(-1)^{n+q-1}\left(n_{+}\left(g_{-\alpha}\right)-n_{-}\left(g_{-\alpha}\right)\right),
$$


where $n_{+}\left(g_{-\alpha}\right)$ (resp. $\left.n_{-}\left(g_{-\alpha}\right)\right)$ is the number of non-degenerate critical points with even (resp. odd) index of $g_{\mid F_{\delta}(g \leq-\alpha)}$. Now we perturbe $g$ into $\tilde{g}$ in a neighborhood of $F_{\delta}(g=0) \cap B_{\varepsilon / 2}$ in such a way that $\tilde{g}_{\mid F_{\delta}}$ is a Morse function near $F_{\delta}(g=0)$. Then we will have

$$
\operatorname{deg} H=\sum_{s \in H^{-1}(0, \delta, 0) /|g(s)|>\alpha} J(s)+\sum_{l} \operatorname{deg}_{q_{\imath}} H(\tilde{g}, f, h),
$$

where the points $q_{i}$ are the critical points of $\tilde{g}_{\mid F_{\delta}}$ lying near $\{g=0\}$. We conclude as in the previous theorem.

Remark 4.5. - When $p=0$, condition (C) is trivially satisfied.

- When $q=2, \Sigma=Z(m)$ and condition (C) is clearly useless.

\section{A formula for the link in dimension 2,4 and 8}

In this section, we study the case $p=n=0$. We consider a coordinate system $\left(y_{1}, \ldots, y_{q}\right), q=2,4,8$, of $\boldsymbol{R}^{q}$. and we define

$$
H: \boldsymbol{R}^{q} \rightarrow \boldsymbol{R}^{q}, y \mapsto(f, m),
$$

where $m$ is defined by formula (1), with $m_{i j}=\partial(g, f) / \partial\left(y_{i}, y_{j}\right), 1 \leq i, j \leq q$. Keeping the previous notations, we get

THEOREM 5.1. Let $\delta$ be a small regular value of $f$ and let $\alpha$ such that $|\alpha| \ll|\delta|$ and $(\delta, \alpha)$ is a regular value of $(f, g)$. If 0 is isolated in $H^{-1}(0)$ then

$$
-\operatorname{deg} H=\chi\left(F_{\delta}(g \geq \alpha)\right)-\chi\left(F_{\delta}(g \leq \alpha)\right)
$$

Proof. In Lemma 3.6 the relation between $\operatorname{Hess}(G)$ and $\bar{J}$ at a Morse critical point $s$ of $g_{\mid F_{\delta}}$ becomes

$$
\operatorname{Hess}(G)=\left(-\frac{1}{f_{x_{1}}^{q}} \bar{J}\right) .
$$

Hence sign $\operatorname{Hess}(G)=-\operatorname{sign}(J)$ at $s$. It is enough to use this relation and the proof of Theorem 4.4 .

As a consequence, we get

THEOREM 5.2. If 0 is isolated in $H^{-1}(0)$ then

$$
\chi\left(f^{-1}(0) \cap\{g \geq 0\} \cap S_{\varepsilon}\right)-\chi\left(f^{-1}(0) \cap\{g \leq 0\} \cap S_{\varepsilon}\right)=-2 \operatorname{deg} H,
$$

where $S_{\varepsilon}$ is the sphere of radius $\varepsilon$.

Proof. Consider the manifold with corners $M=f^{-1}(\delta) \cap\{g \geq \alpha\} \cap B_{\varepsilon}$ where $\delta$ and $\alpha$ are chosen as in the previous theorem. Let 


$$
\partial M=\left(f^{-1}(\delta) \cap\{g \geq \alpha\} \cap S_{\varepsilon}\right) \cup\left(f^{-1}(\delta) \cap g^{-1}(\alpha) \cap B_{\varepsilon}\right) .
$$

Smoothing the corners, there exists a compact manifold with boundary $\tilde{M}$ such that $\operatorname{dim} M=\operatorname{dim} \tilde{M}$ and $(M, \partial M)$ is diffeomorphic to $(\tilde{M}, \partial \tilde{M})$. Hence $\chi(\tilde{M})=$ $\chi(M)$ and $\chi(\partial \tilde{M})=\chi(\partial M)$. But $\tilde{M}$ is odd-dimensional so $\chi(\partial \tilde{M})=2 \chi(\tilde{M})$ and then $\chi(\partial M)=2 \chi(M)$.

Now, by Mayer-Vietoris Sequence,

$$
\begin{aligned}
\chi(\partial M)= & \chi\left(f^{-1}(\delta) \cap\{g \geq \alpha\} \cap S_{\varepsilon}\right)+\chi\left(f^{-1}(\delta) \cap g^{-1}(\alpha) \cap B_{\varepsilon}\right) \\
& -\chi\left(f^{-1}(\delta) \cap g^{-1}(\alpha) \cap S_{\varepsilon}\right) .
\end{aligned}
$$

The latter Euler characteristic vanishes since $f^{-1}(\delta) \cap g^{-1}(\alpha) \cap S_{\varepsilon}$ is compact, odddimensional and smooth for sufficiently small $\delta$ and $\alpha$. So we get that

$$
2 \chi\left(f^{-1}(\delta) \cap\{g \geq \alpha\} \cap B_{\varepsilon}\right)=\chi\left(f^{-1}(\delta) \cap\{g \geq \alpha\} \cap S_{\varepsilon}\right)+\chi\left(f^{-1}(\delta) \cap g^{-1}(\alpha) \cap B_{\varepsilon}\right) .
$$

Similarly, we see that

$$
2 \chi\left(f^{-1}(\delta) \cap\{g \leq \alpha\} \cap B_{\varepsilon}\right)=\chi\left(f^{-1}(\delta) \cap\{g \leq \alpha\} \cap S_{\varepsilon}\right)+\chi\left(f^{-1}(\delta) \cap g^{-1}(\alpha) \cap B_{\varepsilon}\right),
$$

so, by Theorem 5.1,

$$
-2 \operatorname{deg} H=\chi\left(f^{-1}(\delta) \cap\{g \geq \alpha\} \cap S_{\varepsilon}\right)-\chi\left(f^{-1}(\delta) \cap\{g \leq \alpha\} \cap S_{\varepsilon}\right) .
$$

Now Lemma 4.1 tells us that $f^{-1}(0), g^{-1}(0)$ and $S_{\varepsilon}$ intersect transversally so, since $\delta$ and $\alpha$ are sufficiently close to 0 , we obtain that

$$
\chi\left(f^{-1}(0) \cap\{g \geq 0\} \cap S_{\varepsilon}\right)-\chi\left(f^{-1}(0) \cap\{g \leq 0\} \cap S_{\varepsilon}\right)=-2 \operatorname{deg} H .
$$

\section{Examples}

Here are some examples.

\subsection{Example 1.}

Let $f, h, g: \boldsymbol{R}^{6} \rightarrow \boldsymbol{R}$ be defined in the following way

$$
\begin{gathered}
f\left(x, y_{1}, y_{2}, z_{1}, z_{2}, z_{3}\right)=z_{1}^{2}+z_{2}^{2}+z_{3}^{2}+x^{2}+y_{1} z_{2}+y_{2} z_{3}, \\
h\left(x, y_{1}, y_{2}, z_{1}, z_{2}, z_{3}\right)=y_{1} y_{2} \\
g\left(x, y_{1}, y_{2}, z_{1}, z_{2}, z_{3}\right)=y_{1}+y_{2} .
\end{gathered}
$$

Let $\delta>0$ be such that $-\delta$ is a regular value of $f$. We show that $(-\delta, 0)$ is a regular value of $(f, h)$. The Jacobian matrix of $(f, h)$ is

$$
\left(\begin{array}{cccccc}
2 x & z_{2} & z_{3} & 2 z_{1} & 2 z_{2}+y_{1} & 2 z_{3}+y_{2} \\
0 & y_{2} & y_{1} & 0 & 0 & 0
\end{array}\right) .
$$

Let $p$ be a point in $f^{-1}(-\delta) \cap h^{-1}(0)$. If $y_{1} z_{2}-y_{2} z_{3}=0$ at $p$ then, since $y_{1} y_{2}=0$ at $p, f(p)=z_{1}^{2}+z_{2}^{2}+z_{3}^{2}+x^{2} \geq 0$, which is impossible. Thus if $p$ 
belongs to $f^{-1}(-\delta) \cap h^{-1}(0)$ then $y_{1} z_{2}-y_{2} z_{3} \neq 0$ and $(-\delta, 0)$ is a regular value of $(f, h)$.

Now $f^{-1}(-\delta) \cap h^{-1}(0) \cap g^{-1}(0)$ is empty because if $p$ belongs to this intersection then $y_{1}=-y_{2}=0$ and $f(p) \geq 0$, which is impossible.

Let $H=\left(f, h, \partial(g, f, h) / \partial\left(x, y_{1}, y_{2}\right) ; f_{z_{1}}, f_{z_{2}}, f_{z_{3}}\right)$. We have

$$
\begin{aligned}
H\left(x, y_{1}, y_{2}, z_{1}, z_{2}, z_{3}\right)= & \left(z_{1}^{2}+z_{2}^{2}+z_{3}^{2}+x^{2}+y_{1} z_{2}+y_{2} z_{3}, y_{1} y_{2}, 2 x\left(y_{2}-y_{1}\right),\right. \\
& \left.2 z_{1}, 2 z_{2}+y_{1}, 2 z_{3}+y_{2}\right) .
\end{aligned}
$$

It is easy to see that 0 is isolated in $H^{-1}(0)$ and we can apply Theorem 4.3 and get

$$
\operatorname{deg} H=-\left\{\chi\left(F_{-\delta}\left(y_{1}+y_{2} \geq 0\right)\right)-\chi\left(F_{-\delta}\left(y_{1}+y_{2} \leq 0\right)\right)\right\} .
$$

Let us compute $\operatorname{deg} H$. We have

$$
J(H)=\left|\begin{array}{cccccc}
2 x & z_{2} & z_{3} & 2 z_{1} & 2 z_{2}+y_{1} & 2 z_{3}+y_{2} \\
0 & y_{2} & y_{1} & 0 & 0 & 0 \\
2\left(y_{2}-y_{1}\right) & -2 x & 2 x & 0 & 0 & 0 \\
0 & 0 & 0 & 2 & 0 & 0 \\
0 & 1 & 0 & 0 & 2 & 0 \\
0 & 0 & 1 & 0 & 0 & 2
\end{array}\right|
$$

An easy computation gives

$$
J(H)=32 x^{2}\left(y_{2}+y_{1}\right)+8\left(y_{2}-y_{1}\right)\left(-y_{1}^{2}+y_{2}^{2}\right) .
$$

Let $\varepsilon=(0,0, \eta, 0,0,0)$ with $\eta>0$. Then $p \in H^{-1}(\varepsilon)$ if and only if

$$
\begin{gathered}
z_{1}^{2}+z_{2}^{2}+z_{3}^{2}+x^{2}+y_{1} z_{2}+y_{2} z_{3}=0, \\
y_{1} y_{2}=0, \\
2 x\left(y_{2}-y_{1}\right)=\eta, \\
2 z_{1}=0, \\
2 z_{2}+y_{1}=0, \\
2 z_{3}+y_{2}=0 .
\end{gathered}
$$

If $y_{1}=0$ then $z_{2}=0$ and $2 x y_{2}=\eta$. Moreover $z_{3}=-y_{2} / 2$ and $z_{3}^{2}+y_{2} z_{3}=$ $-y_{2}^{2} / 4$. Hence $x^{2}-y_{2}^{2} / 4=0$ which implies that $x= \pm y_{2} / 2$. Since $\eta>0$, we have $x=y_{2} / 2$ and $y_{2}^{2}=\eta$. Finally we have found that

$$
H^{-1}(\varepsilon) \cap\left\{y_{1}=0\right\}=\left\{\left(\frac{\sqrt{\eta}}{2}, 0, \sqrt{\eta}, 0,0,0\right) ;\left(-\frac{\sqrt{\eta}}{2}, 0,-\sqrt{\eta}, 0,0,0\right)\right\} .
$$

Similarly we find

$$
H^{-1}(\varepsilon) \cap\left\{y_{2}=0\right\}=\left\{\left(-\frac{\sqrt{\eta}}{2}, \sqrt{\eta}, 0,0,0,0\right) ;\left(\frac{\sqrt{\eta}}{2},-\sqrt{\eta}, 0,0,0,0\right)\right\} .
$$


It is easy to see that $\varepsilon$ is a regular value of $H$ and that $\operatorname{deg} H=0$. Hence

$$
\chi\left(F_{-\delta}\left(y_{1}+y_{2} \geq 0\right)\right)-\chi\left(F_{-\delta}\left(y_{1}+y_{2} \leq 0\right)\right)=0 .
$$

\subsection{Example 2.}

Keep $f$ and $h$ as in the first example. Let $g=x^{2}$. It is clear that when $g=0, \nabla g=0$ and $(-\delta, 0,0)$ is not a regular value of $(f, h, g)$.

Let $H=\left(f, h, \partial(g, f, h) / \partial\left(x, y_{1}, y_{2}\right) ; f_{z_{1}}, f_{z_{2}}, f_{z_{3}}\right)$. We have

$$
\begin{aligned}
H\left(x, y_{1}, y_{2}, z_{1}, z_{2}, z_{3}\right)= & \left(z_{1}^{2}+z_{2}^{2}+z_{3}^{2}+x^{2}+y_{1} z_{2}+\dot{y}_{2} z_{3}, y_{1} y_{2}, 2 x\left(y_{1} z_{2}-y_{2} z_{3}\right),\right. \\
& \left.2 z_{1}, 2 z_{2}+y_{1}, 2 z_{3}+y_{2}\right) .
\end{aligned}
$$

It is easy to see that 0 is isolated in $H^{-1}(0)$ and we can apply Theorem 4.4 and get

$$
\operatorname{deg} H=-\left\{\chi\left(F_{-\delta}\left(x^{2} \geq 0\right)\right)-\chi\left(F_{-\delta}\left(x^{2} \leq 0\right)\right)\right\}+\mu_{(-\delta, 0)} .
$$

Let us compute $\operatorname{deg} H$. We have

$$
J(H)=\left|\begin{array}{cccccc}
2 x & z_{2} & z_{3} & 2 z_{1} & 2 z_{2}+y_{1} & 2 z_{3}+y_{2} \\
0 & y_{2} & y_{1} & 0 & 0 & 0 \\
2\left(y_{1} z_{2}-y_{2} z_{3}\right) & 2 x z_{2} & -2 x z_{3} & 0 & 2 x y_{1} & -2 x y_{2} \\
0 & 0 & 0 & 2 & 0 & 0 \\
0 & 1 & 0 & 0 & 2 & 0 \\
0 & 0 & 1 & 0 & 0 & 2
\end{array}\right| .
$$

An easy computation gives

$$
J(H)=16 x^{2}\left(y_{2}^{2}-2 y_{2} z_{3}+y_{1}^{2}-2 y_{1} z_{2}\right)+8\left(y_{1} z_{2}-y_{2} z_{3}\right)\left(-y_{1}^{2}+y_{2}^{2}\right) .
$$

Let $\varepsilon=(\alpha, \beta, 0,0,0,0)$ with $\alpha, \beta>0$. Then $p \in H^{-1}(\varepsilon)$ if and only if

$$
\begin{gathered}
z_{1}^{2}+z_{2}^{2}+z_{3}^{2}+x^{2}+y_{1} z_{2}+y_{2} z_{3}=\alpha, \\
y_{1} y_{2}=\beta, \\
2 x\left(y_{1} z_{2}-y_{2} z_{3}\right)=0, \\
2 z_{1}=0, \\
2 z_{2}+y_{1}=0, \\
2 z_{3}+y_{2}=0 .
\end{gathered}
$$

If $x=0$ then, since $z_{2}=-y_{1} / 2$ and $z_{3}=-y_{2} / 2$, we get $-y_{1}^{2}-y_{2}^{2}=4 \alpha$, which is impossible because $\alpha>0$. So if $p \in H^{-1}(\varepsilon)$ then $x \neq 0$ and $y_{1} z_{2}-y_{2} z_{3}=0$. Since $z_{2}=-y_{1} / 2$ and $z_{3}=-y_{2} / 2$, we obtain $y_{1}^{2}=y_{2}^{2}$. But $\beta>0$ and $y_{1}$ and $y_{2}$ have the same sign. Finally we find that $y_{1}=y_{2}= \pm \sqrt{\beta}, x^{2}=\alpha+\beta / 2$, which we write $x^{2}=\gamma$, and 


$$
\begin{aligned}
H^{-1}(\varepsilon)= & \left\{\left(\sqrt{\gamma}, \sqrt{\beta}, \sqrt{\beta}, 0,-\frac{\sqrt{\beta}}{2},-\frac{\sqrt{\beta}}{2}\right) ;\left(-\sqrt{\gamma}, \sqrt{\beta}, \sqrt{\beta}, 0,-\frac{\sqrt{\beta}}{2},-\frac{\sqrt{\beta}}{2}\right) ;\right. \\
& \left.\left(\sqrt{\gamma},-\sqrt{\beta},-\sqrt{\beta}, 0, \frac{\sqrt{\beta}}{2}, \frac{\sqrt{\beta}}{2}\right) ;\left(-\sqrt{\gamma},-\sqrt{\beta},-\sqrt{\beta}, 0, \frac{\sqrt{\beta}}{2}, \frac{\sqrt{\beta}}{2}\right)\right\} .
\end{aligned}
$$

Now it is easy to see that $\varepsilon$ is a regular value of $H$ and that $\operatorname{deg} H=4$. Thus we find

$$
-\chi\left(F_{-\delta}\right)+\chi\left(F_{-\delta}(x=0)\right)+\mu_{(-\delta, 0)}=4 .
$$

\subsection{Example 3.}

Let $f\left(y_{1}, y_{2}, y_{3}, y_{4}\right)=y_{1}^{2}+y_{2}^{2}+y_{3}^{2}-y_{4}^{2}$ and $g\left(y_{1}, y_{2}, y_{3}, y_{4}\right)=y_{1} y_{2}+y_{3} y_{4}$. Let $H$ be defined as in Section 5. We have

$$
H\left(y_{1}, y_{2}, y_{3}, y_{4}\right)=\left(y_{1}^{2}+y_{2}^{2}+y_{3}^{2}-y_{4}^{2},-2 y_{1}^{2}+2 y_{2}^{2}-2 y_{3}^{2}-2 y_{4}^{2}, 4 y_{2} y_{3},-4 y_{2} y_{4}\right) \text {. }
$$

Clearly $H^{-1}(0)=0$. Furthermore $\operatorname{deg} H=0$ because $H^{-1}(0, \beta, 0,0)=\emptyset$ if $\beta>0$ and Theorem 5.2 gives

$$
\chi\left(f^{-1}(0) \cap\{g \geq 0\} \cap S_{\varepsilon}^{3}\right)-\chi\left(f^{-1}(0) \cap\{g \leq 0\} \cap S_{\varepsilon}^{3}\right)=0
$$

where $S_{\varepsilon}^{3}$ is the 3 -dimensional sphere with radius $\varepsilon$.

\section{REFERENCES}

[AFS] K. Aoki, T. FukUda AND W Z. Sun, On the number of branches of a plane curve germ, Kodal Math. J., 9 (1986), 179-187.

[AFN1] K. AOKI, T. FukUdA AND T. Nishimura, On the number of branches of the zero locus of a map germ $\left(\boldsymbol{R}^{n}, 0\right) \rightarrow\left(\boldsymbol{R}^{n-1}, 0\right)$. Topology and Computer Science (S. Suzukı ed.), Kinokuniya, 1987, 347-363.

[AFN2] K. Aoki, T. FukUda AND T. Nishimura, An algebraic formula for the topological types of one parameter bifurcations diagrams, Arch. Rational Mech. Anal., 108 (1989), 247-265.

[Ar] V I. ARNold, Index of a singular point of a vector field, the Petrovsk1-Oleınik inequality, and mixed Hodge structures, Funct. Anal. Appl., 12 (1978), 1-14.

[Dal] J. DAmON, On the number of branches for real and complex weighted homogeneous curve singularities, Topology, 30 (1991), 223-229.

[Da2] J. Damon, $G$-signature, $G$-degree, and symmetries of the branches of curve singularities, Topology, 30 (1991), 565-590.

[Da3] J. DAMON, Equivariant bifurcations and Morsifications for finite groups, Lecture Notes in Math., 1463, Springer-Verlag (1991), 80-106.

[DLNS] P Dudzinski, A. Lecki, P Nowak-Przygodzki and Z. Szafraniec, On the topological invariance of the Milnor number mod 2, Topology, 32 (1993), 573-576.

[Dut1] N. Dutertre, On $p$-parameter of an $n$-dimensional function-germ, Manuscripta Math., 94 (1997), 21-31.

[Dut2] N. Dutertre, About the Milnor fiber of a real map-germ, Prépublication de l'Institut de Recherche Mathématıque de Rennes, 99-04 (1999). 
[Ei] D. EISENBud, An algebraic approach to the topological degree of a smooth map, Bull. Amer. Math. Soc., 84 (1978), 751-764.

[EL] D. EISENBUd AND H. I. LEvine, An algebraic formula for the degree of a $C^{\infty}$ mapgerm, Ann. of Math., 106 (1977), 19-44.

[Fu1] T. FukUI, Mapping degree formula for 2-parameter bifurcation of function-germs, Topology, 32 (1993), 567-571.

[Fu2] T. FukuI, An algebraic fomula for a topological invariant of bifurcation of 1-parameter family of function-germs, Stratifications, Singularitıes, and Differential Equations, II, (D. Trotman ed.) Travaux en Cours 55, Hermann, 1997, 45-54.

[Gr] G. M. GreuEL, Der Gauss-Manın Zusammenhang 1solierter Singularitäten von vollständingen Durschnitten, Math. Ann., 214 (1975), 235-266.

[Ha] H. HАмм, Lokale topologische Eigenschaften komplexer Raume, Math. Ann., 191 (1971), 235-252.

[HL] H. Hamm and Le Dung Trang, Un théorème de Zarıskı du type de Lefschetz, Ann. Scı. Ecol. Norm. Sup. (3), 6 (1973), 317-355.

[Kh] G. M. Khimshiashvili, On the local degree of a smooth map, Soobshch. Akad. Nauk Gruz. SSR, 85 (1977), 309-311.

[Le] Le Dung Trang, Calcul du nombre de Milnor d'une singularité isolée d'intersection complète, Funct. Anal. Appl., 8 (1974), 45-52.

[Mi1] J. Milnor, Morse Theory, Ann Math. Stud., 51, Princeton University Press, 1963.

[Mi2] J. Milnor, Singular Points of Complex Hypersurfaces, Ann Math. Stud., 61, Princeton University Press, 1968.

[MvS] J. Montaldi AND D. Van Straten, One-forms on singular curves and the topology of real curve singularities, Topology, 29 (1990), 501-510.

[Mu T. Muir, A Treatise on the Theory of Determinants, revised edition, Dover, 1960.

[Szl] Z. SzAFraniec, On the number of branches of a 1-dimensional semianalytic set, Koda1 Math. J., 11 (1988), 78-85.

[Sz2] Z. SzAFraniec, A formula for the Euler characteristic of a real algebraic manifold, Manuscripta Math., 85 (1994), 345-360.

[Sz3] Z. SzAFraniEC, On topological invariants of real analytic singularities, in preprint.

[Wa] C. T. C. WALL, Topological invariance of the Milnor number mod 2, Topology, 22 (1983), 345-350.

IMR, UNIVERSITÉ DE RENNES 1

Campus de Beaulieu 35042 RenNes

FRANCE

E-mail: dutertre@maths.unıv-rennes1.fr 
